\title{
Orthobiologics: Diagnosis and Treatment of Common Tendinopathies
}

\author{
Katherine D. Van Schaik, MD, PhD, $\mathrm{MA}^{1}$ Kenneth S. Lee, $\mathrm{MD}^{2,30}$ \\ ${ }^{1}$ Department of Radiology, Beth Israel Deaconess Medical Center, \\ Harvard Medical School, Boston, Massachusetts \\ 2 University of Wisconsin School of Medicine and Public Health, \\ Madison, Wisconsin \\ ${ }^{3}$ Department of Radiology, University of Wisconsin School of \\ Medicine and Public Health, Madison, Wisconsin

\begin{abstract}
Address for correspondence Kenneth S. Lee, MD, University of Wisconsin School of Medicine and Public Health, Madison, WI (e-mail: klee2@uwhealth.org).
\end{abstract} \\ Semin Musculoskelet Radiol 2021;25:735-744.
}

\begin{abstract}
Keywords

- orthobiologics

- platelet-rich plasma

- tendinopathy

- ultrasonography

Orthobiologics, including platelet-rich plasma, prolotherapy, and mesenchymal stem cells, are seeing increasing use in the treatment of osteoarthritis (OA), muscle injury, and tendinopathy. This article reviews the biology and applications of orthobiologics in tendons, muscles, and joints, and focuses on platelet-rich plasma (PRP). Clinical evidence-based literature related to the use of PRP in the treatment of rotator cuff injury, lateral epicondylosis, Achilles tendinopathy, plantar fasciitis, knee OA, and acute muscle injury are discussed.
\end{abstract}

\section{Orthobiologics: An Overview}

The term orthobiologics describes biological agents applied to musculoskeletal (MSK) injuries, most frequently in the form of ultrasonography (US)-guided injection, to reduce pain and to facilitate healing. ${ }^{1,2}$ Such agents include bone grafts, platelet-rich plasma (PRP) (both leukocyte-rich [LR] plasma and leukocyte-poor [LP] plasma), autologous blood, and stem cells (mesenchymal and embryonic). These substances are used to treat a variety of MSK problems, such as tendinopathies, osteoarthritis (OA), and acute muscle injuries, and their use is becoming increasingly relevant for both young and older age groups. MSK pathologies, including arthritis, joint complaints, and traumatic injuries, are rising in prevalence in the United States, especially as the population ages. The most recent edition of the United States Bone and Joint Initiative: The Burden of Musculoskeletal Diseases in the United States estimates that, for MSK disorders, "expenditures in 2014 dollars increased from $\$ 381.4$ billion in 1996-1998 to $\$ 882.5$ billion in 2012-2014, an increase of more than $130 \%$. . . In 1996-1998, aggregate all-cause expenditures for persons with a musculoskeletal disease ... represented $3.2 \%$ of the GDP [gross domestic product]. By 2012-2014, the proportion had grown to 5.2\% of the GDP."3 Orthobiologics are especially important in pain manage- ment as patients and providers alike seek long-term solutions that avoid opiate-based therapies.

The initial theoretical foundation for the use of orthobiologics derived from recognition of the limited regenerative capabilities of the MSK system. It was hypothesized that the injection of growth factors, typically derived from a patient's own blood, would enhance regeneration and repair. Since initial studies in the 1990s demonstrating that platelet-rich plasma (PRP) could hasten the consolidation of mandibular bone grafts, ${ }^{4}$ the use and applications of orthobiologics generally, and PRP particularly, have only continued to expand. This review article focuses on PRP, including its biology and practical applications, with special attention given to the role of the radiologist in the use of this expanding therapy.

\section{Platelet-rich Plasma: Biology and Classification Systems}

PRP, as the name suggests, is a preparation of autologous blood enhanced with an increased concentration of platelets. It is obtained through the centrifugation of anticoagulated whole blood, yielding a plasma layer that is platelet poor, a leukocyte layer that is platelet rich, and a red blood cell (RBC) layer. ${ }^{5-8}$
Issue Theme Musculoskeletal Intervention; Guest Editors, Connie Y. Chang, MD and Mary K. Jesse, MD (c) 2021. Thieme. All rights reserved. Thieme Medical Publishers, Inc., 333 Seventh Avenue, 18th Floor, New York, NY 10001, USA
DOI https://doi.org/ 10.1055/s-0041-1735475. ISSN 1089-7860. 
Table 1 Platelet-rich plasma classification by Ehrenfest et al ${ }^{7}$

\begin{tabular}{|l|l|}
\hline Type & Constituents \\
\hline P-PRP, also called leukocyte-poor platelet-rich plasma & Without leukocytes and with low-density fibrin network after activation \\
\hline L-PRP & With leukocytes and with low-density fibrin network after activation \\
\hline P-PRF, also called leukocyte-poor platelet-rich fibrin & Without leukocytes, with high-density fibrin network \\
\hline L-PRF & With leukocytes, with high-density fibrin network \\
\hline
\end{tabular}

Abbreviations: L-PRF, leukocyte- and platelet-rich fibrin; L-PRP, leukocyte- and platelet-rich plasma; P-PRF, pure platelet-rich fibrin; P-PRP, pure plateletrich plasma.

Platelets, the namesake component of PRP and an important element of the clotting cascade, also play a significant role in tissue healing, a process that is relatively compromised in ligaments, tendons, and joints, given their relatively poor blood supply. ${ }^{8}$ When tissue incurs trauma, a three-phase process of wound healing begins. With the formation of a hematoma in the inflammatory phase, recruited platelets release the growth factors stored in their $\alpha$ granules, including basic fibroblast growth factor, epidermal growth factor, insulinlike growth factor-1, platelet-derived growth factor, transforming growth factor- $\beta$, and vascular endothelial growth factor. ${ }^{5,6,9}$ These factors have a variety of functions, including mitogenesis of fibroblasts, osteoblasts, other growth factors, smooth muscle cells, mesenchymal cells, chondrocytes, and osteoblasts. In the proliferative phase that occurs a few days later, these elements contribute to collagen deposition, angiogenesis, granulation tissue formation, and ultimately wound contraction. The final remodeling phase, during which collagen matures and excess cells undergo apoptosis, can last for months.

In addition to platelets, PRP also contains leukocytes and RBCs. Although leukocytes (including neutrophils, macrophages, and monocytes) are essential for the inflammatory phase of wound healing and help prevent infection, the increased inflammation they promote can counteract the benefits provided by PRP. ${ }^{10-13}$ Recent research has indicated that reducing leukocyte concentrations in PRP, rather than increasing platelet concentrations, may be a crucial aspect of enhancing therapeutic efficacy. ${ }^{10}$ In general, LR-PRP is used to promote inflammation and healing for tendinopathies, whereas LP-PRP is preferred for treating knee OA. Similarly, RBCs are also believed to compromise the efficacy of PRP preparations through the release of cytotoxic oxygen-free radicals in the setting of oxidative stress. ${ }^{14}$ Differing concentrations of each of these components in PRP preparations can make comparisons across research trials challenging. ${ }^{6}$ Still further variability can be introduced into PRP preparations through activation with mixtures of thrombin and calcium that facilitate rapid delivery of growth factors. However, less is known regarding possible benefits of rapid versus gradual delivery of growth factors, with some studies suggesting that rapid delivery enhances bony regeneration but slows fibroblast differentiation. ${ }^{6,15,16}$

The broad term platelet-rich plasma, therefore, can often gloss over differences between subcategories of this substance, which are important to maintain in light of growing evidence that certain concentrations of platelets and growth factors are more suitable for some conditions than others. ${ }^{2}$ Multiple classification systems have been used, each placing variable emphasis upon, for example, concentrations of constituents in the centrifugate, fibrin levels, and/or use of activation techniques, such as adding calcium to promote degranulation of platelets to release growth factors. ${ }^{17}$

The 2014 classification by Ehrenfest et al is widely cited (summarized in - Table 1) and uses the presence or absence of leukocytes, and high- versus low-density fibrin networks, as the basis for categorization. ${ }^{7}$ The 2012 classification system by Mishra et al, also divided into four groups, is based on relative leukocyte levels (increased over baseline versus minimal to no leukocytes) and whether or not the preparation is activated. Each group is subclassified into " $\mathrm{A}$ " (more than five times the baseline level of platelets) or "B" (less than five times the baseline level of platelets). ${ }^{18}$ DeLong et al proposed the platelet-activation-white blood cell (PAW) system, dividing platelet levels into four groups, total white blood cells (WBCs) into two groups, and neutrophil levels into two groups, and providing an additional marker to indicate whether the preparation has undergone activation (-Table 2). ${ }^{19}$ The 2015 platelet count, leukocyte content including percentage of neutrophils if applicable, RBC content, and activation (PLRA) system devised by Mautner et al is both a means of classification and a proposed mechanism for standardized reporting in methodological descriptions. ${ }^{17}$

Such diversity of preparation, description, and reporting creates challenges for the interpretation of PRP studies and

Table 2 PAW classification system by DeLong et al ${ }^{19}$

\begin{tabular}{|l|l|l|}
\hline Platelets & Concentration, platelets/ $\mu \mathrm{L}$ & Label \\
\hline & Baseline or lower & P1 \\
\hline & Above baseline to 750,000 & P2 \\
\hline & $>750,000-1,250,000$ & P3 \\
\hline & $>1,250,000$ & P4 \\
\hline Activation & Exogenous activation applied & $\mathrm{X}$ \\
\hline $\begin{array}{l}\text { White } \\
\text { blood cells }\end{array}$ & WBCs above baseline & $\mathrm{A}$ \\
\hline & WBCs below or equal to baseline & $\mathrm{B}$ \\
\hline & Neutrophils above baseline & $\alpha$ \\
\hline & Neutrophils below baseline & $\beta$ \\
\hline
\end{tabular}

Abbreviations: PAW, platelet-activation white blood cell; WBCs, white blood cells. 
therefore the standardization of PRP therapy. In this review, evidence related to the use of variable preparations is discussed further within each anatomically focused subsection.

\section{Platelet-rich Plasma and Radiology}

Imaging is an essential component in the administration of PRP therapy and in the assessment of its efficacy. Because US guidance is often used in the acquisition of PRP, and nearly always used in its application, radiologists are at the forefront of PRP-based therapies. ${ }^{8,20}$ US also makes PRP therapies portable: because basic preparation of PRP only requires a small tabletop centrifuge and the patient's own blood, injections can occur in operating rooms, radiology suites, sports medicine clinics, or even at sporting events. ${ }^{8}$ - Table 1 Le et al summarize specifications related to commercially available PRP centrifuge systems. ${ }^{21}$ For most systems, $50 \mathrm{~mL}$ of venous blood is required (up to a maximum of $54 \mathrm{~mL}$ ), which can yield an increase in platelets up to 10 times above baseline for LR preparations and up to 7 times above baseline for LP preparations. ${ }^{22}$

For the radiologist performing a PRP injection, the preprocedure protocol is similar to the protocol for other USguided procedures. Before the procedure, the radiologist or practitioner should obtain informed consent from the patient, which involves a discussion of the benefits and the risks that include hemorrhage, tissue injury, and infection (minimized using a sterile technique). Because PRP is considered experimental and therefore often not reimbursed by insurance, a detailed discussion on alternative reimbursable treatments (such as dry needling) should also be considered. Indications for PRP include pain at least 4 of 10 on the visual analog scale (VAS), for at least 3 months, that has not responded to conservative measures such as nonsteroidal anti-inflammatory drugs (NSAIDs) and physical therapy (PT); clinical and imaging findings consistent with tendinopathy, OA, or MSK injuries for which PRP is recommended; ability to follow through with postprocedural restrictions and rehabilitation protocols; and a desire to defer or avoid more invasive therapies. ${ }^{23}$ Contraindications to PRP therapy include the presence of a joint prosthesis, active infection, immunocompromised individual, coagulopathy (including medical anticoagulation), an international normalized ratio $>2.5$, tendon tear, and severely advanced OA. ${ }^{6}$ If the patient is an athlete, she or he must be able to refrain from play for at least 4 to 8 weeks following treatment.

After the blood is obtained and prepared according to the protocols most relevant to the injection site and related pathology, it is injected, typically under US guidance, via a 10 -mL closed-system syringe and a $20 \mathrm{G}$ or $22 \mathrm{G}$ needle. After the injection, patients can expect inflammation that may be associated with an initial increase in pain or discomfort that typically subsides within 24 to 48 hours. NSAIDs should be avoided 2 weeks before and after the injection because their anti-inflammatory properties may compromise the healing benefits provided by the platelets and their associated growth factors. Steroids, too, should be avoided during this time. ${ }^{8}$ Detailed descriptions of, and timelines associated with, postprocedural restrictions and rehabilitation protocols were published by Emory University and the University of Wisconsin Health and are summarized in Wu et al., $6,24,25$

Magnetic resonance imaging (MRI) is used before the procedure to assess candidates' appropriateness for treatment and afterward to evaluate treatment response. MRI assessment before injection enables detailed anatomical characterization of the affected area and can assist with confirming the clinical diagnosis. ${ }^{6}$ Preprocedural imaging also provides a baseline that can be used to evaluate response to therapy. US or computed tomography may also be used in the initial diagnostic work-up, but regardless of the initial modality used, the radiologist will play a central role in preprocedural planning and determination of the suitability of PRP therapy for a given patient. MRI can also be an important component of postprocedural imaging. ${ }^{26-28}$ Post-PRP injection MRI at 3 to 6 months may show improvement, with decreased tendon thickening, as well as decreased T2-weighted signal abnormality and surrounding soft tissue edema typically seen with tendinopathy. Normalization of the tendon structure may also be seen on MRI. Postprocedure US at 3 to 6 months may also show decreased tendon thickening, as well as improvement in the tendon fibrillar pattern, including decreased hypoechogenicity and hyperemia. An innovative US-based technology called shear wave elastography shows promise as a quantitative measure using shear wave speed (meters per second) as a biomarker for tendon healing.

\section{Tendinopathies Commonly Treated with PRP}

As previously described, PRP and its many formulations have demonstrated variable efficacy in different anatomical areas and in the treatment of different pathologies. This section focuses on four of the most common and extensively researched uses of PRP in the treatment of tendinopathy, listed anatomically from upper to lower extremity.

\section{Rotator Cuff Tendinopathy}

Studies of PRP injection for the treatment of rotator cuff tendinopathy have suggested that PRP provides some benefit for up to 1 year (-Fig. 1). A 2020 meta-analysis of five randomized controlled trials (RCTs), ${ }^{29-34}$ comparing PRP injection with sham injection, no injection, or PT alone, found that patients with PRP experienced a reduction in their pain after 24 weeks, although no significant differences in pain between the PRP and other treatment groups was observed at the short (3 weeks) or medium (12 weeks) postprocedural time points. Maximum follow-up was 1 year for three studies ${ }^{29,31,33}$ and 6 months for the other two. ${ }^{30,32}$ Standardized mean difference analysis indicated no significant difference between PRP injection groups and other treatment groups with respect to functional improvement at any postprocedural time point. It should be noted that, in four of these studies, more than one PRP injection was given during the study duration. ${ }^{29,30,32,33}$ Little is known about the number of PRP injections required for optimal tendon treatment, but typically only one PRP injection is 

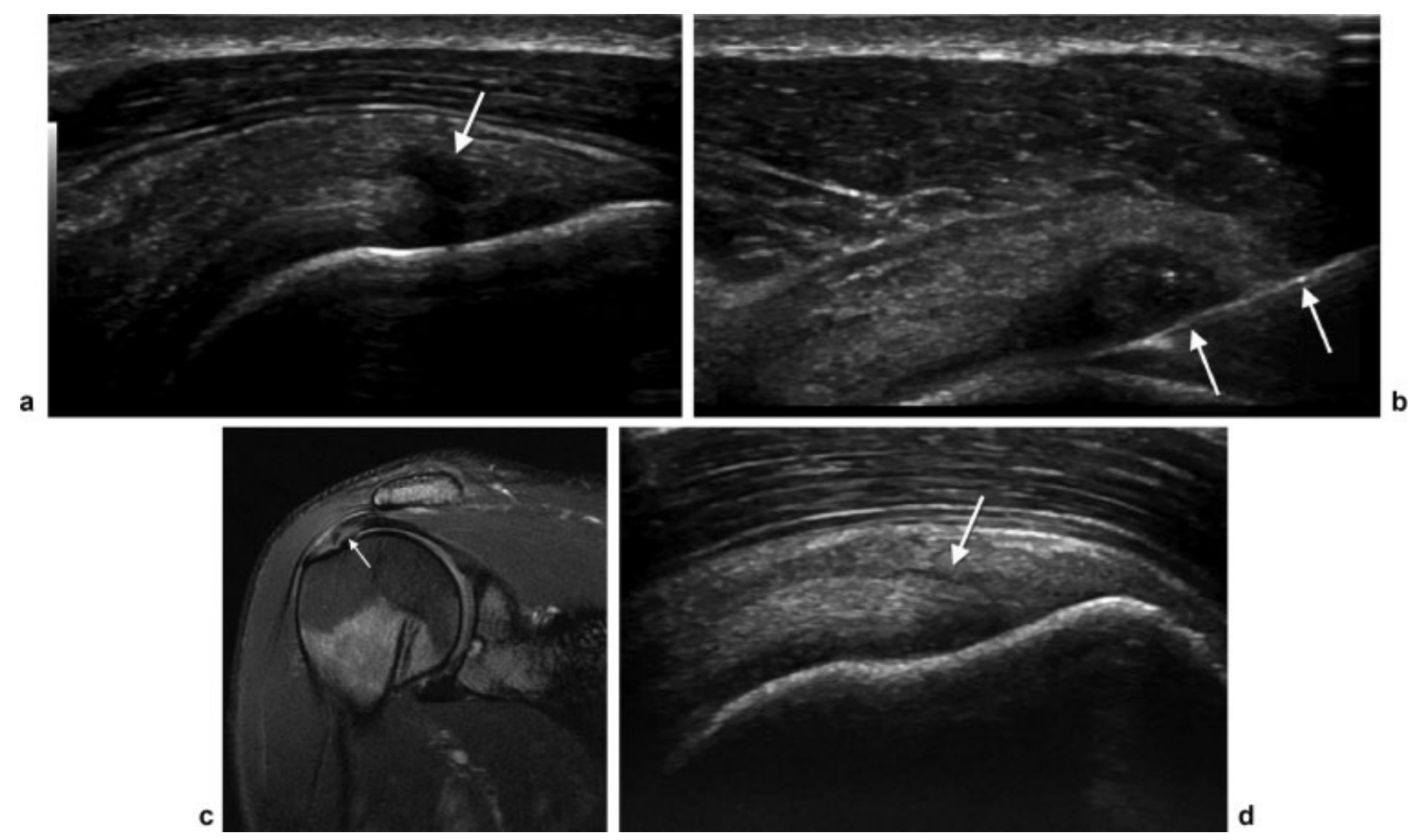

Fig. 1 (a) A 37-year-old woman with shoulder pain. Longitudinal sonogram of the supraspinatus shows focal area of intrasubstance hypoechogenicity suggestive of an interstitial tear (arrow). (b) A 37-year-old woman with shoulder pain. Longitudinal sonogram of the supraspinatus shows a 22G needle (arrows) placed within the focal area of intrasubstance hypoechogenicity during platelet-rich plasma (PRP) treatment. (c) A 37-year-old woman with shoulder pain. Coronal T2-weighted fat-saturated magnetic resonance imaging of the shoulder demonstrates small area of intrasubstance high signal (arrow) corresponding to the ultrasound abnormality seen. (d) A 37-year-old woman 4 months after PRP treatment. Longitudinal sonogram of the supraspinatus shows near-complete resolution of the focal area of intrasubstance hypoechogenicity (arrow) suggestive of healing. Patient's pain and function improved.

performed. Of particular relevance for the radiologist is that four studies used US-guided injection ${ }^{29-32}$ and that $\mathrm{US}^{30}$ or MRI was an integral part of the initial diagnostic work-up and/or follow-up assessment. $29,31,32$

Two RCTs published since this 2020 meta-analysis also showed reductions in pain with administration of PRP injection. A double-blind RCT comparing PRP with corticosteroid injection in 99 patients showed statistically significant improvement in VAS pain scores, American Shoulder and Elbow Surgeon Index scores, and Western Ontario Rotator Cuff Index scores for the PRP group at 3 months after the procedure. However, no differences were observed between the PRP and corticosteroid groups at 12 months. ${ }^{35}$ US or MRI was used to establish preprocedural diagnoses of tendinopathy, and US guidance was used for the injections. A RCT comparing USguided PRP and PT in 64 patients with clinically diagnosed adhesive capsulitis showed no difference in VAS pain scores or in passive range of shoulder motion at 1,3 , and 6 weeks posttreatment. The number of patients taking acetaminophen at each time point was lower for the PRP group. ${ }^{36}$

Although all of these studies described the commercial system used to prepare the PRP and the volume of PRP injected, only Nejati et al described the plasma in detail, noting the platelet concentration $(900,000 \pm 15,000$ platelets $/ \mathrm{mm}^{3}$ ), its magnitude above baseline blood platelet count, and leukocyte concentration $\left(5,000-10,000 / \mathrm{mm}^{3}\right)$. Kwong et al described their preparation as leukocyte poor, with " $80 \%$ platelets at $1.6 \times$ concentration," and noted the manufacturer's reported filtration rates for RBCs, WBCs, mononuclear cells, and granulocytes. Inconsistences in the preparation, administration, and published description of PRP were also noted in a 2018 meta-analysis of six studies assessing PRP for rotator cuff pathologies. This additional meta-analysis showed decreased pain scores for PRP-treated patients at 6.5 months and beyond. ${ }^{37}$ In sum, it is difficult to reproduce these studies and to interpret their results, given the lack of detailed information about the preparation of the PRP that was ultimately administered.

PRP has also been studied as an adjunct to surgical rotator cuff repair. A 2020 meta-analysis of 13 RCTs compared the effect of arthroscopic rotator cuff repair with either LR- or LPPRP on rates of retear, healing, and pain scores. ${ }^{38}$ LP-PRP reduced rates of retear and/or incomplete tendon healing after fixation for both small and medium to large tears. In addition, patients with this treatment experienced reduced pain, as measured by multiple pain scales. However, post hoc analyses showed that LR-PRP did not lead to significant improvements over the control group with respect to any outcome metric. ${ }^{38}$ Durability of therapeutic benefit is not well characterized, with some studies showing lower rates of re-tears at 1 year. ${ }^{39-41}$ Others suggest that improvements in pain and functionality wane after 1 year. ${ }^{41-44}$

\section{Lateral Epicondylosis}

Literature assessing the role of PRP in the treatment of lateral epicondylosis showed clear reductions in pain, especially in 


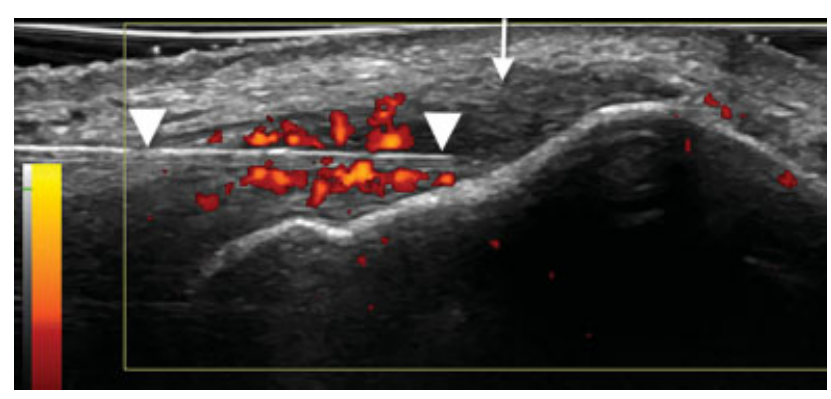

Fig. 2 A 55-year-old man with lateral elbow pain. Longitudinal sonogram of the common extensor tendon (arrow) of the lateral elbow shows thickening and hypoechogenicity. Power Doppler shows hyperemia in red. A $22 \mathrm{G}$ needle (arrowheads) is inserted into the tendon during platelet-rich plasma treatment.

comparison with corticosteroids (-Fig. 2). A 2018 literature review and meta-analysis including 11 articles examining PRP in the treatment of lateral epicondylosis showed no differences in pain scores between PRP and control groups at 2 to 6.5 months postprocedure, but significant reductions in pain scores for the PRP-treated groups at $\geq 6.5$ months postprocedure. ${ }^{37,45-54}$ A prominent and consistent finding in studies examining PRP in the treatment of lateral epicondylosis has been the relatively extended duration of pain reduction, ${ }^{55,56}$ with at least one study demonstrating persistent pain reduction up to 2 years. ${ }^{53}$ PRP was also shown to improve function, as evaluated by both subjective (Disabilities of the Arm, Shoulder, and Hand questionnaire) and objective scales (Mayo Clinic performance index for the elbow). ${ }^{48,49,53,56}$ Comparison between PRP and whole-blood injections revealed improved scores on the Patient-related Tennis Elbow Evaluation (assessment of both pain and subjective function) at both PRP and whole blood. ${ }^{52}$

Some studies focused on lateral epicondylosis showed that PRP injections are not superior to other treatment strategies. For example, a smaller double-blind RCT comparing PRP and saline found decreased pain scores at 6 months for both groups but no statistically significant differences in pain scores between the PRP and control groups. ${ }^{46}$ Another study, which compared PRP, saline, and glucocorticoid injections, found that glucocorticoids reduced color Doppler activity and tendon thickness relative to PRP and saline, and that saline showed better pain reduction at 3 months compared with PRP and glucocorticoids. ${ }^{51}$ A RCT comparing PRP and whole blood found improved pain scores for PRP at only 6 weeks. ${ }^{54}$ A second RCT also comparing PRP and whole blood showed improvements in pain and functionality scores at $1,2,6$, and 12 months but no statistically significant difference between the two injection types. ${ }^{50}$

Imaging is an essential component of the preprocedural diagnostic work-up and the assessment of treatment efficacy for lateral epicondylosis. US was frequently used for preprocedural imaging, injection guidance, and follow-up assessments. ${ }^{49}$ Although MRI was used less frequently in lateral epicondylosis studies, some investigators still employed it in both preprocedural assessments and follow-up evaluations. $^{57}$ In one study, PRP was used as an adjuvant to tenotomy, with improvements in both pain scales and func- tionality for at least 6 months. ${ }^{56}$ A retrospective case review showed that PRP injections reduced the number of patients needing surgery, with an absolute risk reduction of 0.773 and a number needed to treat of 1.3. This study also showed improvement in symptoms with PRP. ${ }^{57}$

Most studies did not characterize the constituents of the PRP injected, aside from mentioning manufacturer details for the separation system. Those that did characterize PRP used preparations without leukocytes and with moderate enrichment of platelets. ${ }^{48,50,56}$ At least one study mentioned platelet concentration but did not discuss leukocyte concentration. ${ }^{51}$ These gaps in reporting the precise features of the PRP used in the studies, a persistent issue for PRP research, regardless of the anatomical site involved, also make research about the role of PRP in the treatment of lateral epicondylosis difficult to interpret.

\section{Patellar Tendinopathy}

Studies regarding the use of PRP in the treatment of patellar tendinopathy have also yielded mixed results. One study showed functional improvements lasting up to 4 years ( - Fig. 3 shows a sonogram of PRP injection into the patellar tendon). ${ }^{58}$ A 2015 review and meta-analysis evaluated 11 studies, of which only two were RCTs. ${ }^{59}$ Among the eight noncomparative studies, four reported on patients' ability to return to activity: rates for pain-free return to activity ranged from $22 \%{ }^{60}$ to $81 \%{ }^{58}$ Comparative studies investigated PRP versus dry needling and exercise (23 patients total) ${ }^{61}$; PRP plus PT versus shock wave therapy plus PT (46 patients total) 62. or PRP versus PT alone (15 patients in the PRP group). ${ }^{58}$ Compared with dry needling, PRP patients had greater improvements in pain at 12 weeks, but the pain returned at 6 months. ${ }^{61}$ In comparison with shock wave therapy, PRP provided greater improvements in pain at 2,6 , and 12 months. PRP versus PT alone showed no significant difference in pain scores. Increased number of injections may contribute to improved outcomes, ${ }^{63}$ but the precise number and chronological pacing of the injections is still poorly characterized. Typically, a single injection of PRP is performed.

Simultaneous injection of PRP and high volumes of saline, which may improve symptoms of patellar tendinopathy by disrupting neurovascular structures and lysing adhesions, ${ }^{64}$ may have more prolonged benefits than injection of saline

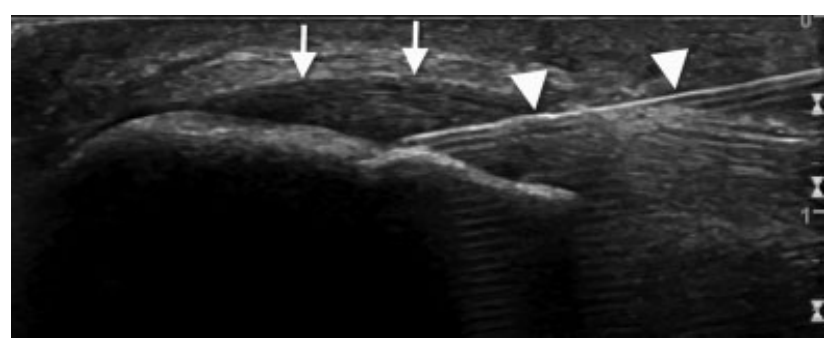

Fig. 3 A 20-year-old man with anterior knee pain below his patella. Longitudinal sonogram of the patellar tendon shows thickening of the proximal patellar tendon (arrows). A 22G needle (arrowheads) is inserted into the tendon from an inferior to superior approach during platelet-rich plasma treatment. 
alone. ${ }^{65}$ Intraoperative PRP, applied in the setting of bonepatellar tendon-bone autograft anterior cruciate ligament reconstruction, provided no significant difference with respect to reductions in pain or functionality when compared with controls. Both groups showed similarly improved symptoms at the same time points, as well as similar MRI findings. ${ }^{66}$ Authors noted that they used LP-PRP with a platelet concentration 2 to 3 times above baseline.

Imaging was discussed less frequently in articles describing PRP therapy for patellar tendinopathy. Diagnosis of patellar tendinopathy was more frequently made clinically, with relatively limited use of diagnostic or postprocedural US. MRI was used to assess postprocedural healing in a few studies, ${ }^{61}$ particularly those using intraoperative PRP. ${ }^{66}$ US guidance was still often used for injection, although its use was described in research articles less frequently for patellar tendon injections than for those in the upper extremity. For example, at least three studies did not use US guidance in PRP injections but instead performed blindly by palpation. ${ }^{58,60,67}$ Consistent with much of the PRP research that has been discussed, most studies only provided details regarding the manufacturer of the PRP preparation system; few, if any, metrics were provided regarding plasma characteristics.

\section{Achilles Tendinopathy}

The compartmentalized nature of the Achilles tendon creates additional challenges in the interpretation of the therapeutic efficacy of PRP studies because injection into the midsubstance or insertional region can yield different results for functional benefit and pain management. Moreover, as observed in patellar tendinopathy studies, the use of saline as a negative control may be misguided. Injection of saline itself appears to yield therapeutic benefit by the mechanical disruption of tendon fibers and scar tissue with the needle and volume of injectant. Although smaller studies have pointed toward improved functionality, ${ }^{68-71}$ even up to 4 years after the procedure, ${ }^{58}$ RCTs and meta-analyses have been less conclusive ( - Fig. 4 shows a sonogram of the Achilles tendon during PRP treatment).

A recent comprehensive systematic review with network meta-analysis of 29 RCTs evaluated multiple therapies for Achilles tendinopathy, including wait-and-see, placebo injections, high-volume injections, whole-blood injections, pro-

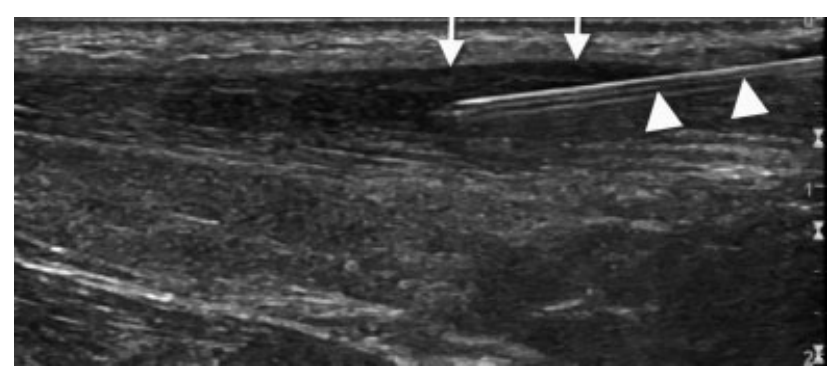

Fig. 4 A 68-year-old woman with posterior ankle pain. Longitudinal sonogram of the Achilles tendon shows thickening (arrows) and hypoechogenicity of the mid-substance Achilles tendon. A 22G needle (arrowheads) is shown from an inferior to superior approach during platelet-rich plasma treatment. lotherapy injections, PRP injections, shock wave therapy, acupuncture, night splinting, mucopolysaccharide supplements, and multiple combinations of these therapies. ${ }^{72}$ All of the included studies were at moderate to high risk for bias. Overall, $86 \%$ of the trials included patients with mid-substance tendinopathy. One-year follow-up showed that injection therapy, exercise alone, exercise and injections, and exercise and night splinting were all comparable in terms of symptom remediation, as measured by the Victorian Institute of Sport Assessment-Achilles score. The authors ultimately concluded that the studies' elevated risk of bias and the large uncertainties in comparative estimates preclude recommendation of a specific treatment. However, the analysis grouped multiple different therapies (e.g., saline injection, prolotherapy, whole-blood injections, and PRP injections) into one intervention group, simply termed "injection therapy," reducing the ability to detect differences in efficacy among these subgroups. Another meta-analysis of five RCTs in 2019 comparing PRP with placebo injections also found that the quality of evidence supplied by these studies was low to moderate. ${ }^{73}$ Statistical analysis of these studies suggested that PRP injection is not superior to saline injections $^{74-77}$ or PT. ${ }^{69}$

US guidance was common for injection protocols, as was the use of both US and MRI to assess the postprocedural appearance of the tendon. ${ }^{68,71,76,78}$

\section{Plantar Fasciitis}

Limited cohort studies have shown promising results for the use of PRP in the setting of plantar fasciitis, with improvement noted in pain, ${ }^{79}$ functionality, ${ }^{80}$ and tissue integrity (-Fig. 5). ${ }^{81}$ Comparisons with corticosteroid injections suggest that PRP is at least as effective as corticosteroids and more effective than saline at pain reduction, 3 months after the injection. ${ }^{82}$ More extended comparative studies demonstrated improvements in pain and functionality up to 2 years. ${ }^{83,84}$ A recent systematic review and meta-analysis included 10 prospective trials and found that PRP therapy provides greater pain relief, compared with corticosteroids, at 3 and 6 months after the injection. ${ }^{85}$ Additional comparisons between PRP and shock wave therapy, ${ }^{86}$ and PRP and prolotherapy, ${ }^{87}$ showed equivalent efficacy regarding pain reduction and improved patient functionality for patients

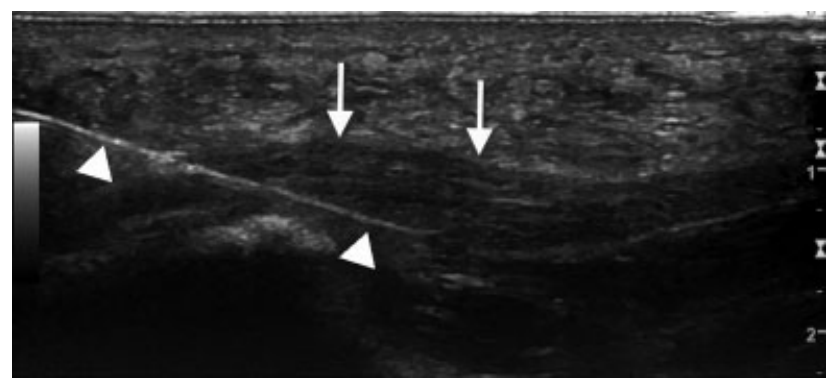

Fig. 5 A 56-year-old man with inferior heel pain. Longitudinal sonogram of the plantar fascia demonstrates thickening (arrows) and hypoechogenicity. A $22 \mathrm{G}$ needle (arrowheads) is inserted from a proximal to distal approach inside the plantar fascia during plateletrich plasma treatment. 
who receive PRP over these other treatments. Imaging (both US and MRI) were used in a few studies for preprocedural evaluation and postprocedural assessment. US-guided injection was used in most studies. PRP preparations were again rarely discussed in detail.

\section{Treatment of Arthritis with PRP: A Brief Note}

PRP is also used to treat other MSK pathologies, including OA. The most extensive research in this area has involved the knee joint. A 2017 systematic review and meta-analysis of 14 RCTs investigating PRP therapy for knee OA yielded equivocal evidence. ${ }^{88-101}$ Four studies were deemed to be of moderate risk for bias and 10 of high risk for bias. ${ }^{102}$ Controls were varied and included saline, hyaluronic acid, ozone, and corticosteroids. PRP was shown to reduce Western Ontario and McMaster Universities Arthritis (WOMAC) pain scores relative to hyaluronic acid and ozone at 6 and 12 months, ${ }^{95}$ and relative to saline at 3,6, and 12 months, ${ }^{98}$ and to improve WOMAC function scores, at 3, 6, and 12 months, postprocedure. However, in a 1-year RCT comparing PRP with viscosupplementation, PRP was not shown to be superior, with diminishing benefit after 9 months. ${ }^{103}$

Reporting of PRP formulations was also rare in these studies, severely limiting evaluation of treatment efficacy and duration.

\section{Conclusions}

In summary, PRP, particularly in its low-leukocyte formulations, has shown promise in the treatment of tendinopathy, especially with respect to lateral epicondylosis. Other studies have shown more limited benefits in rotator cuff, knee, and Achilles tendinopathies. However, recommendations regarding the generation, administration, and assessment of PRP are challenging to make because of substantial heterogeneity in PRP preparations. Future research endeavors should focus on ensuring standardized reporting of PRP components, ideally using one of the classification schemata listed here, to facilitate comparisons across studies.

Conflict of Interest

None declared.

\section{References}

1 Dhillon MS, Behera P, Patel S, Shetty V. Orthobiologics and platelet rich plasma. Indian J Orthop 2014;48(01):1-9

2 Centeno CJ, Pastoriza SM. Past, current, and future interventional orthobiologics techniques and how they relate to regenerative rehabilitation: a clinical commentary. Int J Sports Phys Ther 2020;15(02):301-325

3 United States Bone and Joint Initiative. The burden of musculoskeletal diseases in the United States. 2018. Accessed March 8, 2021 at: boneandjointburden.org

4 Marx RE, Carlson ER, Eichstaedt RM, Schimmele SR, Strauss JE, Georgeff KR. Platelet-rich plasma: growth factor enhancement for bone grafts. Oral Surg Oral Med Oral Pathol Oral Radiol Endod 1998;85(06):638-646
5 Davis VL, Abukabda AB, Radio NM, et al. Platelet-rich preparations to improve healing. Part I: workable options for every size practice. J Oral Implantol 2014;40(04):500-510

6 Wu PI-K, Diaz R, Borg-Stein J. Platelet-rich plasma. Phys Med Rehabil Clin N Am 2016;27(04):825-853

7 Dohan Ehrenfest DM, Andia I, Zumstein MA, Zhang C-Q, Pinto NR, Bielecki T. Classification of platelet concentrates (Platelet-Rich Plasma-PRP, Platelet-Rich Fibrin-PRF) for topical and infiltrative use in orthopedic and sports medicine: current consensus, clinical implications and perspectives. Muscles Ligaments Tendons J 2014;4(01):3-9

8 Lee KS. Ultrasound-guided platelet-rich plasma treatment: application and technique. Semin Musculoskelet Radiol 2016;20 (05):422-431

9 Boswell SG, Cole BJ, Sundman EA, Karas V, Fortier LA. Plateletrich plasma: a milieu of bioactive factors. Arthroscopy 2012;28 (03):429-439

10 Boswell SG, Schnabel LV, Mohammed HO, Sundman EA, Minas T, Fortier LA. Increasing platelet concentrations in leukocyte-reduced platelet-rich plasma decrease collagen gene synthesis in tendons. Am J Sports Med 2014;42(01):42-49

11 Braun HJ, Kim HJ, Chu CR, Dragoo JL. The effect of platelet-rich plasma formulations and blood products on human synoviocytes: implications for intra-articular injury and therapy. Am J Sports Med 2014;42(05):1204-1210

12 McCarrel TM, Minas T, Fortier LA. Optimization of leukocyte concentration in platelet-rich plasma for the treatment of tendinopathy. J Bone Joint Surg Am 2012;94(19):e143, 1-8)

13 Assirelli E, Filardo G, Mariani E, et al. Effect of two different preparations of platelet-rich plasma on synoviocytes. Knee Surg Sports Traumatol Arthrosc 2015;23(09):2690-2703

14 Hooiveld M, Roosendaal G, Wenting M, van den Berg M, Bijlsma J, Lafeber F. Short-term exposure of cartilage to blood results in chondrocyte apoptosis. Am J Pathol 2003;162(03):943-951

15 Roh YH, Kim W, Park KU, Oh JH. Cytokine-release kinetics of platelet-rich plasma according to various activation protocols. Bone Joint Res 2016;5(02):37-45

16 Jeon YR, Jung BK, Roh TS, et al. Comparing the effect of nonactivated platelet-rich plasma, activated platelet-rich plasma, and bone morphogenetic protein-2 on calvarial bone regeneration. J Craniofac Surg 2016;27(02):317-321

17 Mautner K, Malanga GA, Smith J, et al. A call for a standard classification system for future biologic research: the rationale for new PRP nomenclature. PM R 2015;7(4, Suppl):S53-S59

18 Mishra A, Harmon K, Woodall J, Vieira A. Sports medicine applications of platelet rich plasma. Curr Pharm Biotechnol 2012;13(07):1185-1195

19 DeLong JM, Russell RP, Mazzocca AD. Platelet-rich plasma: the PAW classification system. Arthroscopy 2012;28(07):998-1009

20 Malanga G, Abdelshahed D, Jayaram P. Orthobiologic interventions using ultrasound guidance. Phys Med Rehabil Clin N Am 2016;27(03):717-731

21 Le ADK, Enweze L, DeBaun MR, Dragoo JL. Current clinical recommendations for use of platelet-rich plasma. Curr Rev Musculoskelet Med 2018;11(04):624-634

22 Degen RM, Bernard JA, Oliver KS, Dines JS. Commercial separation systems designed for preparation of platelet-rich plasma yield differences in cellular composition. HSS J 2017;13(01): $75-80$

23 Nguyen RT, Borg-Stein J, McInnis K. Applications of platelet-rich plasma in musculoskeletal and sports medicine: an evidencebased approach. PM R 2011;3(03):226-250

24 UW School of Medicine and Public HealthSports medicine postoperative rehabilitation guidelines. Accessed March 8, 2021 at: https://patient.uwhealth.org/education/sports-medicine-rehabilitation-guidelines 
25 Emory Healthcare. Platelet-rich-plasma therapy. Accessed March 8, 2021 at: https://www.emoryhealthcare.org/orthopedics/ platelet-rich-plasma-therapy.html

26 Radice F, Yánez R, Gutiérrez V, Rosales J, Pinedo M, Coda S. Comparison of magnetic resonance imaging findings in anterior cruciate ligament grafts with and without autologous plateletderived growth factors. Arthroscopy 2010;26(01):50-57

27 Rupreht M, Vogrin M, Hussein M. MRI evaluation of tibial tunnel wall cortical bone formation after platelet-rich plasma applied during anterior cruciate ligament reconstruction. Radiol Oncol 2013;47(02):119-124

28 Rupreht M, Jevtič V, Serša I, Vogrin M, Jevšek M. Evaluation of the tibial tunnel after intraoperatively administered platelet-rich plasma gel during anterior cruciate ligament reconstruction using diffusion weighted and dynamic contrast-enhanced MRI. J Magn Reson Imaging 2013;37(04):928-935

29 Cai YU, Sun Z, Liao B, Song Z, Xiao T, Zhu P. Sodium hyaluronate and platelet-rich plasma for partial-thickness rotator cuff tears. Med Sci Sports Exerc 2019;51(02):227-233

30 Rha DW, Park G-Y, Kim Y-K, Kim MT, Lee SC. Comparison of the therapeutic effects of ultrasound-guided platelet-rich plasma injection and dry needling in rotator cuff disease: a randomized controlled trial. Clin Rehabil 2013;27(02):113-122

31 Kesikburun S, Tan AK, Yilmaz B, Yaşar E, Yazicioğlu K. Plateletrich plasma injections in the treatment of chronic rotator cuff tendinopathy: a randomized controlled trial with 1-year followup. Am J Sports Med 2013;41(11):2609-2616

32 Nejati P, Ghahremaninia A, Naderi F, Gharibzadeh S, Mazaherinezhad A. Treatment of subacromial impingement syndrome: platelet-rich plasma or exercise therapy? A randomized controlled trial. Orthop J Sports Med 2017;5(05):2325967117702366

33 Ilhanli I, Guder N, Gul M. Platelet-rich plasma treatment with physical therapy in chronic partial supraspinatus tears. Iran Red Crescent Med J 2015;17(09):e23732

34 Lin M-T, Wei K-C, Wu C-H. Effectiveness of platelet-rich plasma injection in rotator cuff tendinopathy: a systematic review and meta-analysis of randomized controlled trials. Diagnostics (Basel) 2020;10(04):189

35 Kwong CA, Woodmass JM, Gusnowski EM, et al. Platelet-rich plasma in patients with partial-thickness rotator cuff tears or tendinopathy leads to significantly improved short-term pain relief and function compared with corticosteroid injection: a double-blind randomized controlled trial. Arthroscopy 2021;37 (02):510-517

36 Thu AC, Kwak SG, Shein WN, Htun M, Htwe TTH, Chang MC. Comparison of ultrasound-guided platelet-rich plasma injection and conventional physical therapy for management of adhesive capsulitis: a randomized trial. J Int Med Res 2020;48(12): 300060520976032

37 Chen X, Jones IA, Park C, Vangsness CT Jr. The efficacy of plateletrich plasma on tendon and ligament healing: a systematic review and meta-analysis with bias assessment. Am J Sports Med 2018;46(08):2020-2032

38 Hurley ET, Colasanti CA, Anil U, et al. The effect of platelet-rich plasma leukocyte concentration on arthroscopic rotator cuff repair: a network meta-analysis of randomized controlled trials. Am J Sports Med 2021;49(09):2528-2535

39 Gumina S, Campagna V, Ferrazza G, et al. Use of platelet-leukocyte membrane in arthroscopic repair of large rotator cuff tears: a prospective randomized study. J Bone Joint Surg Am 2012;94 (15):1345-1352

40 Randelli P, Arrigoni P, Ragone V, Aliprandi A, Cabitza P. Platelet rich plasma in arthroscopic rotator cuff repair: a prospective RCT study, 2-year follow-up. J Shoulder Elbow Surg 2011;20(04): 518-528

41 Castricini R, Longo UG, De Benedetto $\mathrm{M}$, et al. Platelet-rich plasma augmentation for arthroscopic rotator cuff repair: a randomized controlled trial. Am J Sports Med 2011;39(02): 258-265

42 Antuña S, Barco R, Martínez Diez JM, Sánchez Márquez JM. Platelet-rich fibrin in arthroscopic repair of massive rotator cuff tears: a prospective randomized pilot clinical trial. Acta Orthop Belg 2013;79(01):25-30

43 Rodeo SA, Delos D, Williams RJ, Adler RS, Pearle A, Warren RF. The effect of platelet-rich fibrin matrix on rotator cuff tendon healing: a prospective, randomized clinical study. Am J Sports Med 2012;40(06):1234-1241

44 Malavolta EA, Gracitelli MEC, Ferreira Neto AA, Assunção JH, Bordalo-Rodrigues M, de Camargo OP. Platelet-rich plasma in rotator cuff repair: a prospective randomized study. Am J Sports Med 2014;42(10):2446-2454

45 Lebiedziński R, Synder M, Buchcic P, Polguj M, Grzegorzewski A, Sibiński M. A randomized study of autologous conditioned plasma and steroid injections in the treatment of lateral epicondylitis. Int Orthop 2015;39(11):2199-2203

46 Montalvan B, Le Goux P, Klouche S, Borgel D, Hardy P, Breban M. Inefficacy of ultrasound-guided local injections of autologous conditioned plasma for recent epicondylitis: results of a double-blind placebo-controlled randomized clinical trial with one-year follow-up. Rheumatology (Oxford) 2016;55(02): 279-285

47 Tetschke E, Rudolf M, Lohmann CH, Stärke C. Autologous proliferative therapies in recalcitrant lateral epicondylitis. Am J Phys Med Rehabil 2015;94(09):696-706

48 Behera P, Dhillon M, Aggarwal S, Marwaha N, Prakash M. Leukocyte-poor platelet-rich plasma versus bupivacaine for recalcitrant lateral epicondylar tendinopathy. J Orthop Surg (Hong Kong) 2015;23(01):6-10

49 Gautam VK, Verma S, Batra S, Bhatnagar N, Arora S. Platelet-rich plasma versus corticosteroid injection for recalcitrant lateral epicondylitis: clinical and ultrasonographic evaluation. J Orthop Surg (Hong Kong) 2015;23(01):1-5

50 Raeissadat SA, Rayegani SM, Hassanabadi H, Rahimi R, Sedighipour L, Rostami K. Is Platelet-rich plasma superior to whole blood in the management of chronic tennis elbow: one year randomized clinical trial. BMC Sports Sci Med Rehabil 2014;6(01):12

51 Krogh TP, Fredberg U, Stengaard-Pedersen K, Christensen R, Jensen P, Ellingsen T. Treatment of lateral epicondylitis with platelet-rich plasma, glucocorticoid, or saline: a randomized, double-blind, placebo-controlled trial. Am J Sports Med 2013;41 (03):625-635

52 Creaney L, Wallace A, Curtis M, Connell D. Growth factor-based therapies provide additional benefit beyond physical therapy in resistant elbow tendinopathy: a prospective, single-blind, randomised trial of autologous blood injections versus platelet-rich plasma injections. Br J Sports Med 2011;45(12):966-971

53 Gosens T, Peerbooms JC, van Laar W, den Oudsten BL. Ongoing positive effect of platelet-rich plasma versus corticosteroid injection in lateral epicondylitis: a double-blind randomized controlled trial with 2-year follow-up. Am J Sports Med 2011;39 (06):1200-1208

54 Thanasas C, Papadimitriou G, Charalambidis C, Paraskevopoulos I, Papanikolaou A. Platelet-rich plasma versus autologous whole blood for the treatment of chronic lateral elbow epicondylitis: a randomized controlled clinical trial. Am J Sports Med 2011;39 (10):2130-2134

55 Kia C, Baldino J, Bell R, Ramji A, Uyeki C, Mazzocca A. Platelet-rich plasma: review of current literature on its use for tendon and ligament pathology. Curr Rev Musculoskelet Med 2018;11(04): 566-572

56 Martin JI, Atilano L, Merino J, et al. Platelet-rich plasma versus lidocaine as tenotomy adjuvants in people with elbow epicondylopathy: a randomized controlled trial. J Orthop Surg Res 2019;14(01):109 
57 Hastie G, Soufi M, Wilson J, Roy B. Platelet rich plasma injections for lateral epicondylitis of the elbow reduce the need for surgical intervention. J Orthop 2018;15(01):239-241

58 Filardo G, Kon E, Di Matteo B, Pelotti P, Di Martino A, Marcacci M. Platelet-rich plasma for the treatment of patellar tendinopathy: clinical and imaging findings at medium-term follow-up. Int Orthop 2013;37(08):1583-1589

59 Liddle AD, Rodríguez-Merchán EC. Platelet-rich plasma in the treatment of patellar tendinopathy: a systematic review. Am J Sports Med 2015;43(10):2583-2590

60 Gosens T, Den Oudsten BL, Fievez E, van 't Spijker P, Fievez A. Pain and activity levels before and after platelet-rich plasma injection treatment of patellar tendinopathy: a prospective cohort study and the influence of previous treatments. Int Orthop 2012;36 (09):1941-1946

61 Dragoo JL, Wasterlain AS, Braun HJ, Nead KT. Platelet-rich plasma as a treatment for patellar tendinopathy: a double-blind, randomized controlled trial. Am J Sports Med 2014;42(03):610-618

62 Vetrano M, Castorina A, Vulpiani MC, Baldini R, Pavan A, Ferretti A. Platelet-rich plasma versus focused shock waves in the treatment of jumper's knee in athletes. Am J Sports Med 2013; 41(04):795-803

63 Zayni R, Thaunat M, Fayard J-M, et al. Platelet-rich plasma as a treatment for chronic patellar tendinopathy: comparison of a single versus two consecutive injections. Muscles Ligaments Tendons J 2015;5(02):92-98

64 Maffulli N, Del Buono A, Oliva F, Testa V, Capasso G, Maffulli G. High-volume image-guided injection for recalcitrant patellar tendinopathy in athletes. Clin J Sport Med 2016;26(01):12-16

65 Abate M, Di Carlo L, Verna S, Di Gregorio P, Schiavone C, Salini V. Synergistic activity of platelet rich plasma and high volume image guided injection for patellar tendinopathy. Knee Surg Sports Traumatol Arthrosc 2018;26(12):3645-3651

66 Walters BL, Porter DA, Hobart SJ, et al. Effect of intraoperative platelet-rich plasma treatment on postoperative donor site knee pain in patellar tendon autograft anterior cruciate ligament reconstruction: a double-blind randomized controlled trial. Am J Sports Med 2018;46(08):1827-1835

67 Kon E, Filardo G, Delcogliano M, et al. Platelet-rich plasma: new clinical application: a pilot study for treatment of jumper's knee. Injury 2009;40(06):598-603

68 Oloff L, Elmi E, Nelson J, Crain J. Retrospective analysis of the effectiveness of platelet-rich plasma in the treatment of achilles tendinopathy: pretreatment and posttreatment correlation of magnetic resonance imaging and clinical assessment. Foot Ankle Spec 2015;8(06):490-497

69 Kearney RS, Parsons N, Costa ML. Achilles tendinopathy management: a pilot randomised controlled trial comparing plateletrich plasma injection with an eccentric loading programme. Bone Joint Res 2013;2(10):227-232

70 Murawski CD, Smyth NA, Newman H, Kennedy JG. A single platelet-rich plasma injection for chronic midsubstance Achilles tendinopathy: a retrospective preliminary analysis. Foot Ankle Spec 2014;7(05):372-376

71 Owens RF Jr, Ginnetti J, Conti SF, Latona C. Clinical and magnetic resonance imaging outcomes following platelet rich plasma injection for chronic midsubstance Achilles tendinopathy. Foot Ankle Int 2011;32(11):1032-1039

72 van der Vlist AC, Winters M, Weir A, et al. Which treatment is most effective for patients with Achilles tendinopathy? A living systematic review with network meta-analysis of 29 randomised controlled trials. Br J Sports Med 2021;55(05):249-256

73 Liu CJ, Yu KL, Bai JB, Tian DH, Liu GL. Platelet-rich plasma injection for the treatment of chronic Achilles tendinopathy: a meta-analysis. Medicine (Baltimore) 2019;98(16):e15278

74 de Jonge S, de Vos RJ, Weir A, et al. One-year follow-up of plateletrich plasma treatment in chronic Achilles tendinopathy: a double-blind randomized placebo-controlled trial. Am J Sports Med 2011;39(08):1623-1629

75 de Vos RJ, Weir A, van Schie HTM, et al. Platelet-rich plasma injection for chronic Achilles tendinopathy: a randomized controlled trial. JAMA 2010;303(02):144-149

76 Krogh TP, Ellingsen T, Christensen R, Jensen P, Fredberg U. Ultrasound-guided injection therapy of Achilles tendinopathy with platelet-rich plasma or saline: a randomized, blinded, placebocontrolled trial. Am J Sports Med 2016;44(08):1990-1997

77 Boesen AP, Hansen R, Boesen MI, Malliaras P, Langberg H. Effect of high-volume injection, platelet-rich plasma, and sham treatment in chronic midportion Achilles tendinopathy: a randomized double-blinded prospective study. Am J Sports Med 2017; 45(09):2034-2043

78 Albano D, Messina C, Usuelli FG, et al. Magnetic resonance and ultrasound in achilles tendinopathy: predictive role and response assessment to platelet-rich plasma and adipose-derived stromal vascular fraction injection. Eur J Radiol 2017; 95:130-135

79 Martinelli N, Marinozzi A, Carnì S, Trovato U, Bianchi A, Denaro V. Platelet-rich plasma injections for chronic plantar fasciitis. Int Orthop 2013;37(05):839-842

80 Kumar V, Millar T, Murphy PN, Clough T. The treatment of intractable plantar fasciitis with platelet-rich plasma injection. Foot 2013;23(2-3):74-77

81 Ragab EMS, Othman AMA. Platelets rich plasma for treatment of chronic plantar fasciitis. Arch Orthop Trauma Surg 2012;132 (08):1065-1070

82 Mahindra P, Yamin M, Selhi HS, Singla S, Soni A. Chronic plantar fasciitis: effect of platelet-rich plasma, corticosteroid, and placebo. Orthopedics 2016;39(02):e285-e289

83 Jain K, Murphy PN, Clough TM. Platelet rich plasma versus corticosteroid injection for plantar fasciitis: a comparative study. Foot 2015;25(04):235-237

84 Monto RR. Platelet-rich plasma efficacy versus corticosteroid injection treatment for chronic severe plantar fasciitis. Foot Ankle Int 2014;35(04):313-318

85 Mohammed W, Farah S, Nassiri M, McKenna J. Therapeutic efficacy of platelet-rich plasma injection compared to corticosteroid injection in plantar fasciitis: a systematic review and meta-analysis. J Orthop 2020;22:124-134

86 Chew KTL, Leong D, Lin CY, Lim KK, Tan B. Comparison of autologous conditioned plasma injection, extracorporeal shockwave therapy, and conventional treatment for plantar fasciitis: a randomized trial. PM R 2013;5(12):1035-1043

$87 \mathrm{Kim} \mathrm{E}$, Lee JH. Autologous platelet-rich plasma versus dextrose prolotherapy for the treatment of chronic recalcitrant plantar fasciitis. PM R 2014;6(02):152-158

88 Sánchez M, Fiz N, Azofra J, et al. A randomized clinical trial evaluating plasma rich in growth factors (PRGF-Endoret) versus hyaluronic acid in the short-term treatment of symptomatic knee osteoarthritis. Arthroscopy 2012;28(08):1070-1078

89 Spaková T, Rosocha J, Lacko M, Harvanová D, Gharaibeh A. Treatment of knee joint osteoarthritis with autologous platelet-rich plasma in comparison with hyaluronic acid. Am J Phys Med Rehabil 2012;91(05):411-417

90 Vaquerizo V, Plasencia MÁ, Arribas I, et al. Comparison of intraarticular injections of plasma rich in growth factors (PRGFEndoret) versus Durolane hyaluronic acid in the treatment of patients with symptomatic osteoarthritis: a randomized controlled trial. Arthroscopy 2013;29(10):1635-1643

91 Cerza F, Carnì S, Carcangiu A, et al. Comparison between hyaluronic acid and platelet-rich plasma, intra-articular infiltration in the treatment of gonarthrosis. Am J Sports Med 2012;40(12): 2822-2827

92 Li M, Zhang C, Ai Z, Yuan T, Feng Y, Jia W. Therapeutic effectiveness of intra-knee-articular injection of platelet-rich plasma on 
knee articular cartilage degeneration. [in Chinese]. Zhongguo Xiu Fu Chong Jian Wai Ke Za Zhi 2011;25(10):1192-1196

93 Patel S, Dhillon MS, Aggarwal S, Marwaha N, Jain A. Treatment with platelet-rich plasma is more effective than placebo for knee osteoarthritis: a prospective, double-blind, randomized trial. Am J Sports Med 2013;41(02):356-364

94 Filardo G, Kon E, Di Martino A, et al. Platelet-rich plasma vs hyaluronic acid to treat knee degenerative pathology: study design and preliminary results of a randomized controlled trial. BMC Musculoskelet Disord 2012;13(01):229

95 Duymus TM, Mutlu S, Dernek B, Komur B, Aydogmus S, Kesiktas FN. Choice of intra-articular injection in treatment of knee osteoarthritis: platelet-rich plasma, hyaluronic acid or ozone options. Knee Surg Sports Traumatol Arthrosc 2017;25(02): 485-492

96 Paterson KL, Nicholls M, Bennell KL, Bates D. Intra-articular injection of photo-activated platelet-rich plasma in patients with knee osteoarthritis: a double-blind, randomized controlled pilot study. BMC Musculoskelet Disord 2016;17:67

97 Raeissadat SA, Rayegani SM, Hassanabadi H, et al. Knee osteoarthritis injection choices: platelet- rich plasma (PRP) versus hyaluronic acid (a one-year randomized clinical trial). Clin Med Insights Arthritis Musculoskelet Disord 2015;8:1-8

98 Smith PA. Intra-articular autologous conditioned plasma injections provide safe and efficacious treatment for knee osteoar- thritis: an FDA-sanctioned, randomized, double-blind, placebocontrolled clinical trial. Am J Sports Med 2016;44(04):884-891

99 Montañez-Heredia E, Irízar S, Huertas PJ, et al. Intra-articular injections of platelet-rich plasma versus hyaluronic acid in the treatment of osteoarthritic knee pain: a randomized clinical trial in the context of the Spanish national health care system. Int J Mol Sci 2016;17(07):E1064

100 Forogh B, Mianehsaz E, Shoaee S, Ahadi T, Raissi GR, Sajadi S. Effect of single injection of platelet-rich plasma in comparison with corticosteroid on knee osteoarthritis: a double-blind randomized clinical trial. J Sports Med Phys Fitness 2016;56(7-8): 901-908

101 Görmeli G, Görmeli CA, Ataoglu B, Çolak C, Aslantürk O, Ertem K. Multiple PRP injections are more effective than single injections and hyaluronic acid in knees with early osteoarthritis: a randomized, double-blind, placebo-controlled trial. Knee Surg Sports Traumatol Arthrosc 2017;25(03):958-965

102 Shen L, Yuan T, Chen S, Xie X, Zhang C. The temporal effect of platelet-rich plasma on pain and physical function in the treatment of knee osteoarthritis: systematic review and meta-analysis of randomized controlled trials. J Orthop Surg Res 2017;12(01):16

103 Filardo G, Di Matteo B, Di Martino A, et al. Platelet-rich plasma intra-articular knee injections show no superiority versus viscosupplementation: a randomized controlled trial. Am J Sports Med 2015;43(07):1575-1582 